\section{A) Check for updates}

Cite this: Food Funct., 2022, 13, 3381

\title{
Beneficial effects of eugenol supplementation on gut microbiota and hepatic steatosis in high-fat-fed mice
}

\author{
Marina Rodrigues, (D) *a Caroline Bertoncini-Silva, (D) a \\ Anderson Gregorio Joaquim, (D) a Cássia Dias Machado, ${ }^{a}$ \\ Leandra Náira Zambelli Ramalho, ${ }^{\mathrm{b}}$ Daniela Carlos, ${ }^{\mathrm{c}}$ Priscila Giacomo Fassini ${ }^{\mathrm{a}}$ and \\ Vivian Marques Miguel Suen ${ }^{a}$
}

\begin{abstract}
Due to the increase in the prevalence of obesity, new therapies have emerged and eugenol has been shown to be beneficial in metabolic changes and gut microbiota. This study aimed to investigate the effects of eugenol on gut microbiota, hepatic lipid accumulation, body weight, adipose tissue weight, lipid and glycemic profile in mice fed a high-fat diet. Forty C57BL/6 male mice were divided into standard diet (SD), high-fat diet (HFD), standard diet with eugenol (SDE) and high-fat diet with eugenol (HFDE). The dose used of eugenol was $500 \mathrm{mg} \mathrm{kg}^{-1}$ for 8 weeks. Eugenol did not prevent weight gain, but it was effective in preventing hepatic lipid accumulation evidenced by the presence of fat droplets in the HFD group and absence in the HFDE group. An improvement in the gut microbiota profile was observed, proved by an increase in the Actinobacteria phylum in the treated groups and a reduction of Proteobacteria phylum in the HFDE group. Despite not preventing weight gain, eugenol appeared to have a protective effect on hepatic lipid accumulation and beneficially modulate the gut microbiota in mice fed with HFD.
\end{abstract}

Received 27th October 2021 Accepted 3rd February 2022

DOI: 10.1039/d1fo03619j

rsc.li/food-function and the composition of the GM. ${ }^{10-13}$ The GM is composed mainly of seven phyla: Firmicutes, Bacteroidetes, Proteobacteria, Actinobacteria, Verrucomicrobia, Fusobacteria, and Cyanobacteria, with Firmicutes and Bacteroidetes being the most abundant species. ${ }^{10}$ The imbalance between beneficial and pathogenic bacteria that compose the microbiota is defined as dysbiosis. ${ }^{11,14}$ Lifestyle, diet, immune system, some types of antibiotics, age, and type of delivery are some factors that affect the GM and may contribute to dysbiosis and consequently to the development of many diseases. ${ }^{15-17}$ Studies have shown that the composition of the GM between healthy individuals and individuals with obesity is different, both in humans and in animal models. ${ }^{18,19}$ For example, individuals with obesity have an altered relationship between Bacteroidetes and Firmicutes, characterized by a decrease in the Bacteroidetes phylum and an increase in the Firmicutes phylum, compared to eutrophic individuals. ${ }^{11,20}$

Among the various treatment possibilities for obesity, unconventional therapies can be mentioned. One of these therapies is herbal medicines, which can contribute to satiety and increase energy metabolism and consequently promote weight loss. ${ }^{21}$ Eugenol is the major bioactive component extracted from clove oil ${ }^{22,23}$ and has anti-inflammatory, antimicrobial, antibacterial, antioxidant, antiviral, anesthetic, anti-

\footnotetext{
${ }^{a}$ Department of Internal Medicine, Division of Nutrology, Ribeirão Preto Medical School, University of São Paulo, Avenida Bandeirantes, 3900, Monte Alegre, CEP, 14049-900 Ribeirão Preto, São Paulo, Brazil. E-mail: marina.rnutri@gmail.com ${ }^{b}$ Departament of Pathology, Ribeirão Preto Medical School, University of São Paulo, Avenida Bandeirantes, 3900, Monte Alegre, CEP, 14049-900 Ribeirão Preto, São Paulo, Brazil

${ }^{c}$ Department of Biochemistry and Immunology, Ribeirão Preto Medical School, University of São Paulo, Avenida Bandeirantes, 3900, Monte Alegre, CEP, 14049-900 Ribeirão Preto, São Paulo, Brazil
} 
tumor, antidiabetic, antiseptic, and insecticide effects. ${ }^{23-25}$ Wlodarska et $a .^{26}$ performed a study testing six phytonutrients, including eugenol, to evaluate the mechanism of action and the ability to alter the mucosal immune responses in the intestines of mice. They showed that all phytonutrients promoted changes in the expression of innate immune genes in the colon. However, just eugenol stimulated the production of the inner mucus layer, an important barrier for microbes, suggesting that eugenol acts to strengthen the mucous barrier, which protects against pathogens and invasive diseases.

Therefore, considering the possible effects of eugenol modulating properties of the innate immune system in mouse intestine, and the role of the GM in the pathophysiology of obesity, we explore the effects of eugenol on the modulation of the GM to investigate whether this substance influences the bacterial profile, and verify if any associated changes to GM could be beneficial towards the prevention or treatment of obesity. Thus, this study aimed to investigate the effects of eugenol on gut microbiota, histology of the liver, body and adipose tissue weight in mice fed a high-fat diet.

\section{Materials and methods}

\subsection{Ethics statement}

The experimental protocol was approved by the Animal Research Ethics Committee of Ribeirão Preto Medical School, University of São Paulo (protocol number 016/2015-1) and the ethical principles had their origins in the Declaration of Helsinki in the U.K. Animals (Scientific Procedures) and was in accordance with the ARRIVE guidelines.

\subsection{Animals and treatment}

Forty C57BL/6 male mice, 6-8 weeks old, weighing on average $20 \mathrm{~g}$, were obtained from the Animal Facilities of Ribeirão Preto Medical School of the University of São Paulo, and kept in cages under controlled conditions of $12 \mathrm{~h}$ dark-light cycles and constant temperature $\left(25 \pm 1^{\circ} \mathrm{C}\right)$ with water and diet ad libitum.

The animals were randomly separated into four groups of ten animals each: standard diet (SD), high-fat diet (HFD), standard diet with eugenol (SDE), and high-fat diet with eugenol (HFDE). All groups were fed with an industrialized diet (AIN $93 \mathrm{G}$ ) and the HFD group received $40 \%$ of lipids. The nutritional composition and the ingredients of the standard diet and the high-fat diet are described in Table 1.

Eugenol (97-99\% purity) was purchased from SigmaAldrich (Ref: E5504). Animals from the SDE and HFDE groups received $500 \mathrm{mg} \mathrm{kg}^{-1}$ of body weight per day of eugenol supplementation with soybean oil as a vehicle by gavage. Groups that did not receive supplementation (SD and HFD), received only soy oil by gavage.

The animals were kept in an adaptation period for 3 weeks (pre-treatment) and received supplementation for 8 weeks (treatment period) thus undergoing 11 weeks of the experiment. They were weighed before the beginning of the experi-
Table 1 Nutritional composition of the standard and high-fat diets ${ }^{a}(\mathrm{~g} /$ $1000 \mathrm{~g}$ diet)

\begin{tabular}{lll}
\hline Macronutrients & Standard diet & High-fat diet \\
\hline Carbohydrates & $639.5 \mathrm{~g}(64 \%)$ & $483.7 \mathrm{~g}(41.3 \%)$ \\
Lipids & $70 \mathrm{~g}(15.8 \%)$ & $207 \mathrm{~g}(39.8 \%)$ \\
Proteins & $203 \mathrm{~g}(20.3 \%)$ & $221.8 \mathrm{~g}(18.9 \%)$ \\
Energy & $4000 \mathrm{kcal}$ & $4685.2 \mathrm{kcal}$
\end{tabular}

${ }^{a}$ Source: PragSoluções. ${ }^{b}$ Standard diet (AIN 93G), contains the following nutritional composition: $397.5 \mathrm{~g}$ corn starch; $200 \mathrm{~g}$ casein; $132 \mathrm{~g}$ dextrinized starch; $100 \mathrm{~g}$ sucrose; $70 \mathrm{~g}$ soybean oil; $50 \mathrm{~g}$ microcrystalline cellulose; $35 \mathrm{~g}$ mineral mix; $10 \mathrm{~g}$ vitamin mix; $3 \mathrm{~g}$ L-cystine; $2.5 \mathrm{~g}$ choline bitartrate; $0.014 \mathrm{~g} \mathrm{BHT}{ }^{c}{ }^{c}$ High-fat diet (AIN $93 \mathrm{HF} 40 \%$ ), contains the following nutritional composition: $273.7 \mathrm{~g}$ corn starch; $220 \mathrm{~g}$ casein; $100 \mathrm{~g}$ dextrinized starch; $100 \mathrm{~g}$ sucrose; $40 \mathrm{~g}$ soybean oil; $167 \mathrm{~g}$ lard; $50 \mathrm{~g}$ microcrystalline cellulose; $35 \mathrm{~g}$ mineral mix; $10 \mathrm{~g}$ vitamin mix; $1.8 \mathrm{~g}$ L-cystine; $2.5 \mathrm{~g}$ choline bitartrate; $0.014 \mathrm{~g}$ BHT.

ment and weekly thereafter using a digital scale with a maximum capacity of $15 \mathrm{~kg}$ (Filizola S.A., São Paulo, Brazil).

At the end of the experiment, the animals were euthanized by decapitation and blood samples were collected and promptly centrifuged at $3500 \mathrm{rpm}, 4{ }^{\circ} \mathrm{C}$ for $15 \mathrm{~min}$ to obtain the serum, which was kept in a freezer at $-70{ }^{\circ} \mathrm{C}$ for subsequent biochemical analysis. During the sacrifice, samples of hepatic tissue, pancreas, epididymal, retroperitoneal, and brown adipose tissue were collected and weighed. Liver fragments were separated and fixed in $10 \%$ buffered formalin for $48 \mathrm{~h}$ and embedded in paraffin for histological analysis. Stool samples were collected for gut microbiota analysis and stored in a freezer at $-70^{\circ} \mathrm{C}$ until the analysis.

\subsection{Glucose tolerance test (GTT)}

The glucose tolerance test followed a previously standardized methodology. ${ }^{27,28}$ It was performed after a $10 \mathrm{~h}$ fasting period, along with the first blood collection (time 0 ) by a cut at the caudal end of the animal. Subsequently, a glucose solution of $1 \mathrm{mg} \mathrm{g}^{-1}$ of the animal's weight was injected intraperitoneally. Blood collection was performed at times $0^{\prime}, 30^{\prime}, 60^{\prime}$, and $120^{\prime}$. Glucose concentrations were determined by the Accu-Chek glucometer (Accu-chek Performa Roche, Mannheim, Germany) according to the manufacturer's specifications.

\subsection{Biochemical analysis}

Insulin analysis was performed by the commercial Elisa Ultrasensitive Kit (Mouse Ultrasensitive Insulin ELISA, Alpco Diagnostics, Salem USA). Total cholesterol, triglycerides and HDL were analyzed by enzymatic method using commercial kits from Labtest (Labtest Diagnóstica S.A., Brazil). ${ }^{27,28}$

\subsection{Histological analysis}

Formalin-fixed and paraffin-embedded tissue sections were cut to a thickness of $5 \mu \mathrm{m}$ and stained with hematoxylin and eosin for histological examination. A pathologist who was blinded for the treatment used light microscopy to evaluate liver histological alterations, such as steatosis and the degree of changes: ${ }^{27}$ absence of change, mild, moderate, and severe 
changes. The analysis was performed on 10 randomly chosen high-power fields (HPFs; $\times 40$ magnification) in each slide.

\subsection{Gut microbiota analysis}

To evaluate the relative abundance of phyla in the gut microbiota, a simple fecal collection of fecal pellets was performed, located at the end of the intestine (rectum). The tweezer used to collect these samples was exclusive and separated from the other materials. Fecal samples, were frozen at $-70{ }^{\circ} \mathrm{C}$ until phylum quantitative PCR (q-PCR) analysis.

Fecal DNA was obtained using the DNeasy® PowerSoil ${ }^{\circledR}$ kit (Qiagen, Hilden, Germany). Relative abundance of bacteria from the Firmicutes, Bacteroidetes, Actinobacteria, and Proteobacteria phyla was determined using universal primers for the 16S rRNA gene (Eubacteria) or specific for the different phyla.

The primer sequences are described in Table $2 .{ }^{29}$ The differences $(\Delta \mathrm{CT})$ between the cycle threshold (CT) values of Eubacteria and specific bacterial groups were used to obtain normalized levels of each bacterial group $(2-\Delta \mathrm{CT})$. The relative abundance of each bacterial group was obtained after normalization with the control groups.

\subsection{Statistical analysis}

Sample size was determined using previous experimental studies for metabolic assessment. All data are presented as mean and standard derivation. The Kolmogorov-Smirnov test was adopted to identify the normality of the data. All statistical analyses were performed using a one-way ANOVA, followed by post hoc Tukey's test for multiple comparisons and to identify

Table 2 Sequence of primers of interest in this study

\begin{tabular}{ll}
\hline Primer & Sequência \\
\hline Actinobacteria & FP-TGTAGCGGTGGAATGCGC \\
Bacteroidetes & RP-AATTAAGCCACATGCTCCGCT \\
& FP-GTTTAATTCATGATACGCGAG \\
Firmicutes & RP-TTAASCCGACACCTCACGG \\
& FP-ATGTGGTTAATCGAAGCA \\
Proteobacteria & RP-AGCTGACGACAACCATGCAC \\
& FP-CATGACGTTACCCGCAGAAGAAG \\
Eubacteria universal & RP-CTCTACGAGACTCAAGCTTGC \\
& FP-ACTCCTACGGGAGGCAGCAGT \\
& RP-ATTACCGCGGCTGCTGGC
\end{tabular}

Forward primer (FP), reverse primer (RP). Source: ref. 29. differences between groups. Data analysis was performed using the IBM SPSS version 22 software. A significance level of $5 \%$ was adopted.

\section{Results}

\subsection{Effects of eugenol on body weight and food intake in experimental groups}

No difference was observed for the initial weight among the groups. The final weight and, consequently, the percentage of weight gain were higher in the HFDE group when compared with the SDE group. Relative to food intake, a significant difference was observed between the HFD group when compared to the SD group, since the HFD group presented lower food intake than the SD group, but both groups had a similar weight gain, showing the efficacy of high fat diet with higher energy density (Table 3).

\subsection{Effect of eugenol on tissue weight in experimental groups}

As expected, retroperitoneal and epididymal white adipose tissue weight were significantly higher in HFD groups in comparison with SD groups, showing the effect of a high-fat diet. No difference was observed in the liver, pancreas, and brown adipose tissue weights (Table 4 ).

\subsection{Effect of eugenol on biochemical profile in experimental groups}

In order to evaluate the lipid profile, we detected the total cholesterol in serum and observed that in the HFDE group it was significantly higher than in the HFD group $(45 \%, p<$ 0.05). Furthermore, there was a significant increase in the HDL-c in the HFDE group when compared to the HFD group (73\%, $p<0.05)$, and regarding triglycerides, the HFD group showed significantly lower values $(38 \%, p<0.05)$ than the SD group. No significant statistical differences were observed for insulin levels in the serum between the groups (Table 5).

\subsection{Effect of eugenol on GTT in experimental groups}

As shown in Fig. 1, glycemia was similar in all groups at all time points, except at 30 minutes, when the SD and SDE groups had a $20 \%$ lower blood glucose peak; however it was not statistically significant. In a similar manner, the area under the curve was not different between the groups as well.

Table 3 Body weight gain and food intake of mice fed with standard and high-fat diet with and without eugenol

\begin{tabular}{|c|c|c|c|c|}
\hline & $\mathrm{SD}$ & HFD & SDE & HFDE \\
\hline Initial weight $(\mathrm{g})$ & $20 \pm 1.7$ & $19.5 \pm 1.2$ & $19.7 \pm 0.9$ & $19 \pm 0.8$ \\
\hline Final weight $(\mathrm{g})$ & $28.8 \pm 1.8$ & $28.4 \pm 2.0$ & $26.7 \pm 1.5$ & $28.8 \pm 1.7^{\&}$ \\
\hline Weight gain (\%) & $44.5 \pm 9.4$ & $45.9 \pm 10.2$ & $35.7 \pm 7.2$ & $51.9 \pm 11.6^{8}$ \\
\hline Food intake ( $\mathrm{g}$ per week) & $2.39 \pm 0.06$ & $1.91 \pm 0.07^{*}$ & $2.27 \pm 0.07$ & $2.04 \pm 0.11$ \\
\hline
\end{tabular}

Standard diet (SD), high-fat diet (HFD), standard diet with eugenol (SDE), and high-fat diet with eugenol (HFDE). * Different from SD, ${ }^{\star}$ different from SDE. All the groups were analyzed by one-way ANOVA followed by Tukey's test $(p<0.05)$. Data are represented as mean \pm standard deviation, $n=10$ per group. 
Table 4 Tissue weight of mice fed with standard and high-fat diet with and without eugenol (analysis at the end of experimental period)

\begin{tabular}{|c|c|c|c|c|}
\hline & SD & HFD & SDE & HFDE \\
\hline Retroperitoneal adipose tissue (g) & $0.52 \pm 0.27$ & $0.99 \pm 0.40^{*}$ & $0.46 \pm 0.22$ & $0.74 \pm 0.35$ \\
\hline Epididymal adipose tissue $(\mathrm{g})$ & $1.35 \pm 0.63$ & $2.36 \pm 0.78^{*}$ & $1.53 \pm 0.47$ & $2.24 \pm 0.79$ \\
\hline Brown adipose tissue $(\mathrm{g})$ & $0.34 \pm 0.06$ & $0.32 \pm 0.08$ & $0.36 \pm 0.09$ & $0.33 \pm 0.79$ \\
\hline Liver $(\mathrm{g})$ & $3.97 \pm 0.80$ & $3.58 \pm 0.44$ & $4.41 \pm 0.08$ & $4.13 \pm 0.43$ \\
\hline Pancreas (g) & $0.43 \pm 0.09$ & $0.34 \pm 0.08$ & $0.42 \pm 0.11$ & $0.41 \pm 0.07$ \\
\hline
\end{tabular}

Standard diet (SD), high-fat diet (HFD), standard diet with eugenol (SDE), and high-fat diet with eugenol (HFDE).* Different from SD. All the groups were analyzed by one-way ANOVA followed by Tukey's test $(p<0.05)$. Data are represented as mean \pm standard deviation, $n=10$ per group.

Table 5 Plasma biochemical profile of mice fed with standard and high-fat diet with and without eugenol (analysis at the end of experimental period)

\begin{tabular}{lllll}
\hline & SD & HFD & SDE & HFDE \\
\hline HDL cholesterol $\left(\mathrm{mg} \mathrm{dL}^{-1}\right)$ & $84.5 \pm 26.8$ & $77.6 \pm 14.4$ & $58.6 \pm 30.5$ & $101.5 \pm 13.8^{\&}$ \\
Total cholesterol $\left(\mathrm{mg} \mathrm{dL}^{-1}\right)$ & $121.8 \pm 29.6$ & $123 \pm 27.0$ & $144.1 \pm 47.4$ & $178.1 \pm 28.5^{\#}$ \\
Triglycerides $\left(\mathrm{mg} \mathrm{dL}^{-1}\right)$ & $90.4 \pm 29.2$ & $55.8 \pm 7.2^{*}$ & $81.3 \pm 28.1$ & $61.2 \pm 15.8$ \\
Insulin $\left(\mathrm{ng} \mathrm{dL}^{-1}\right)$ & $0.7 \pm 0.4$ & $0.7 \pm 0.3$ & $0.8 \pm 0.5$
\end{tabular}

Standard diet (SD), high-fat diet (HFD), standard diet with eugenol (SDE), and high-fat diet with eugenol (HFDE). *Different from SD, ${ }^{\#}$ different from HFD, ${ }^{\&}$ different from SDE. All the groups were analyzed by one-way ANOVA followed by Tukey's test $(p<0.05)$. Data are represented as mean \pm standard deviation, $n=10$ per group.

a

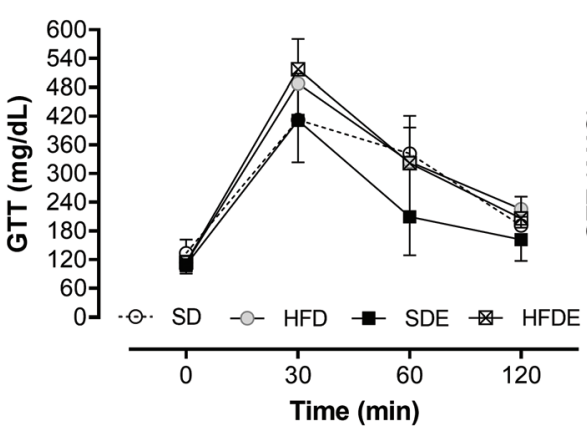

b

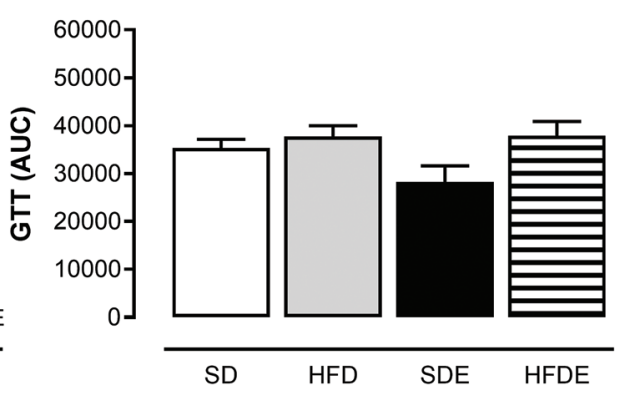

Fig. 1 (a) Glucose tolerance test (GTT) of the experimental groups at the different time points. (b) Area under the curve. Standard diet (SD), high-fat diet (HFD), standard diet with eugenol (SDE), and high-fat diet with eugenol (HFDE). All the groups were analyzed by one-way ANOVA followed by Tukey's test $(p<0.05)$. Data are represented as mean \pm standard deviation, $n=10$ per group.

\subsection{Effect of eugenol on histological analysis of liver in experimental groups}

Fig. 2 shows the histological analysis of the liver of animals from different groups. It was observed that the liver of the animals from the HFD group (Fig. 2b) showed the presence of fat droplets (pointed by the arrow), while in the HFDE group these droplets were absent (Fig. 2d), suggesting that eugenol was effective in preventing fat accumulation in the liver of the mice that received a high-fat diet.

\subsection{Effect of eugenol on gut microbiota composition in experimental groups}

The groups supplemented with eugenol (SDE and HFDE groups) showed an increased relative abundance of the phyla Firmicutes, Bacteroidetes and Actinobacteria (Fig. 3a, $\mathrm{b}$ and $\mathrm{c}$ ). On the other hand, the HFDE group had lower values of Proteobacteria phylum when compared to the SDE group, indicating that eugenol supplementation reduced this phylum in the gut microbiota induced by HFD (Fig. 3d).

\section{Discussion}

The present study demonstrated that eugenol supplementation prevented fat accumulation in the liver of the mice fed with a high-fat diet and promoted an increase in the abundance of Actinobacteria phylum and a reduction in the phylum Proteobacteria. On the other hand, eugenol did not prevent 


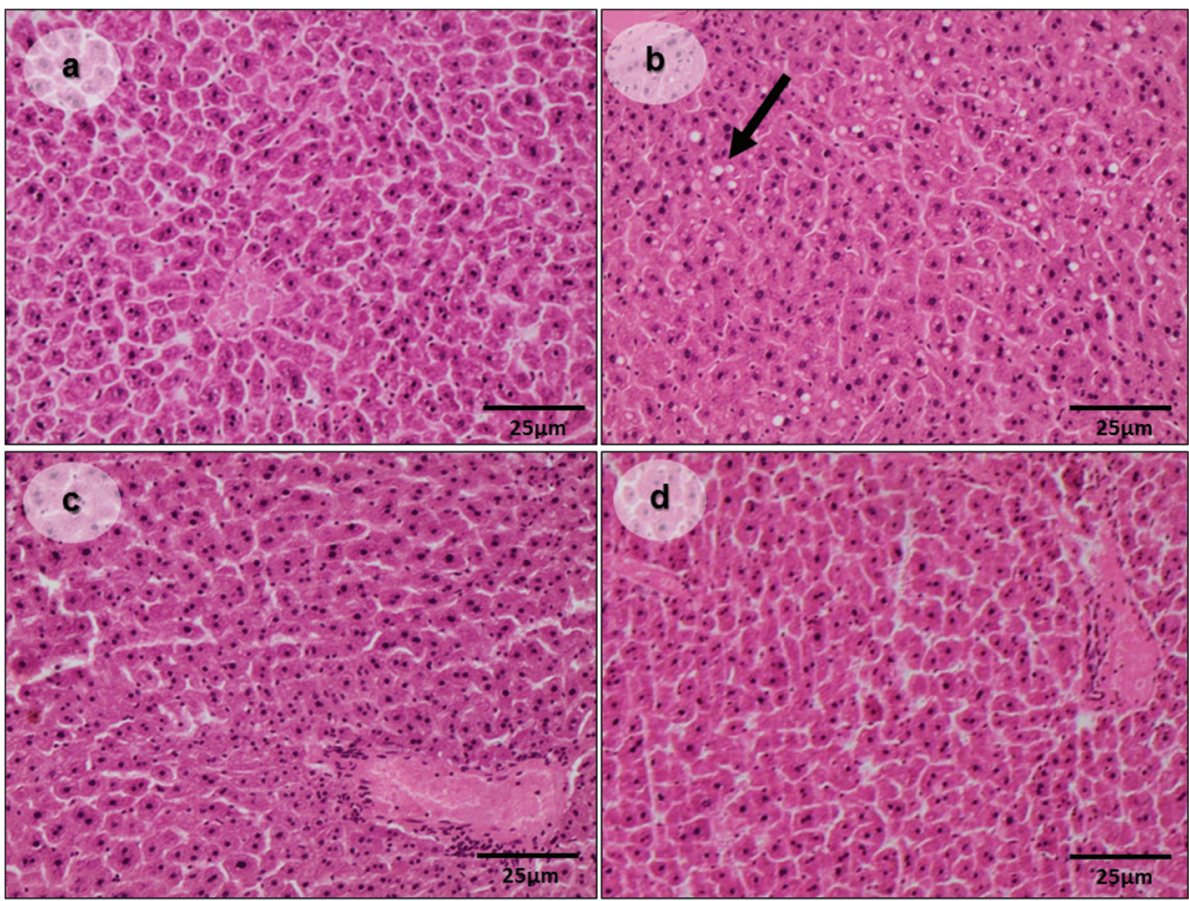

Fig. 2 Histological analysis of the liver. (a) Standard diet (SD); (b) high-fat diet (HFD); (c) standard diet with eugenol (SDE); (d) high-fat diet with eugenol (HFDE). (Hematoxylin and eosin, $\times 40$ magnification).
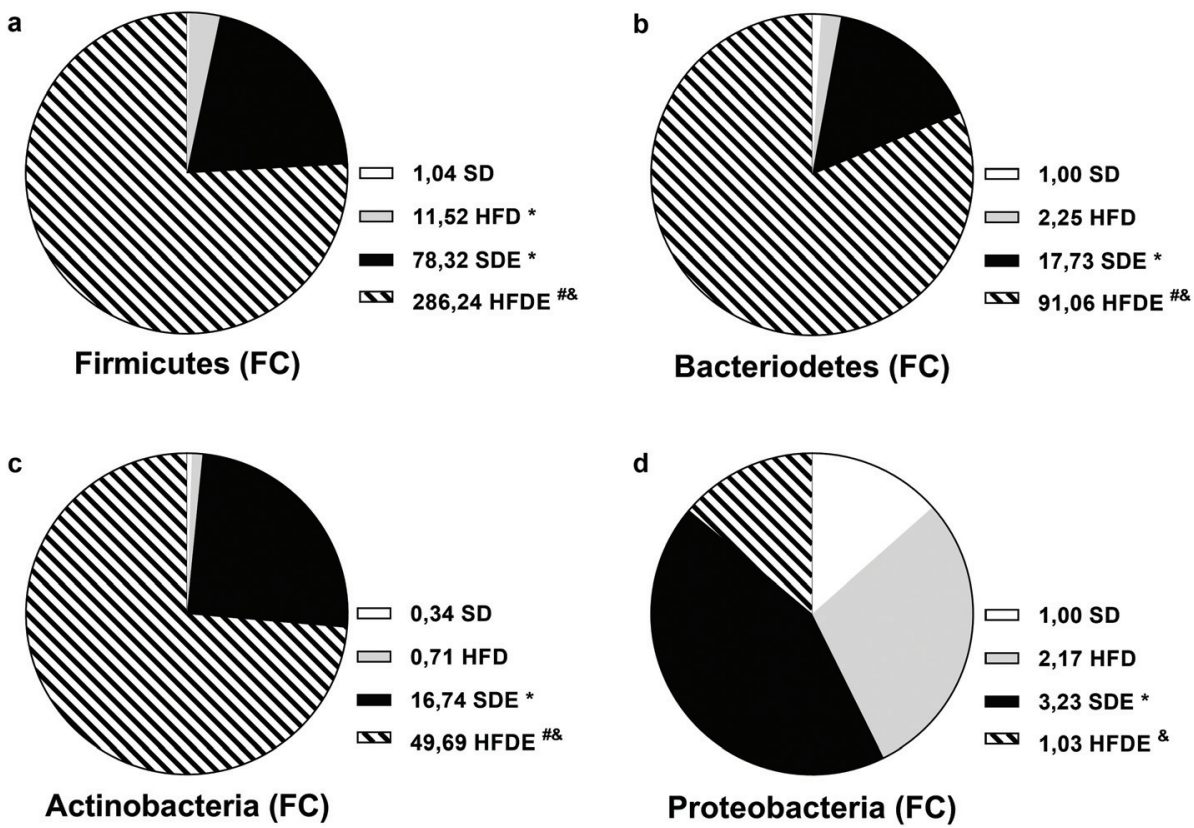

Fig. 3 Relative abundance of phyla of gut microbiota. (a) Firmicutes; (b) Bacteriodetes; (c) Actinobacteria; (d) Proteobacteria. Fold change (FC). Standard diet (SD); high-fat diet (HFD); standard diet with eugenol (SDE); high-fat diet with eugenol. *Different from SD, \#different from HFD, \&different from SDE. All the groups were analyzed by one-way ANOVA followed by Tukey's test $(p<0.05)$. Data are represented as mean \pm standard deviation, $n=10$ per group.

weight gain or increased serum glucose levels, neither increased total cholesterol in mice fed a high-fat diet.

A preliminary study was previously carried out to define the eugenol dose used in the present study. However, the chosen dose is within a non-toxic dose according to De Souza. ${ }^{30}$
Regarding food intake, we observed a difference between the SD and HFD groups. Our findings agreed with White et al., ${ }^{31}$ and although not significantly, the SD and SDE groups had a higher total food intake. Another study using an aspirin ester with eugenol (AEE) in Wistar rats for 8 weeks found that the 
food intake of the HFD and HFD + AEE groups was significantly lower than the SD group, and no difference was observed between the HDF and HFD + AEE groups. ${ }^{32}$

The body weight results showed a difference between SDE and HFDE groups, where the HFDE group showed higher weight gain (40\%). The SD and HFD groups gained equal weight, which explains, the effectiveness of the diet since the HFD group had a lower food intake and gained more weight. This fact is due to the higher energy density of the high-fat diet. Our results differed from Jung et al. and Ding et al., where clove supplementation associated with HFD promoted less body weight gain when compared to HFD only. ${ }^{33,34}$ In this context, Harb et al. used two different doses of eugenol (10 mg and $100 \mathrm{mg}$ per $\mathrm{kg}$ of body weight) in Wistar rats fed a hypercholesterolemic diet (HCD) and standard diet (SD) for 4 weeks. In contrast to the current study, all groups started the experiment with similar body weight, but animals from HCD and HCD + eugenol $100 \mathrm{mg}$ groups gained less weight when compared to those of the SD group. ${ }^{35}$

After analyzing the tissue weight, no differences were found for the liver weight. Our findings diverge from previous studies, since Jung et al. and Ding et al. observed lower liver weight for the high-fat diet treated group compared to the untreated group. ${ }^{33,34}$ Another study supplemented eugenol in C57BL/6J mice fed HFD for 12 weeks and found that eugenol significantly reduced liver weight compared to the untreated HFD group. ${ }^{36}$ These controversial results can be explained by the time of treatment of our study, which was shorter than of other studies. Later, Harb et al. concluded that the groups fed with HCD and HCD + eugenol (10 $\mathrm{mg}$ and $100 \mathrm{mg}$ ) had a higher liver weight than the SD group, and the HCD + eugenol $10 \mathrm{mg}$ group had a significantly lower liver weight than the HCD group. ${ }^{35}$

For adipose tissue weight, Jung et al. and Ding et al. showed that the epididymal adipose tissue weight in the HFD group with clove extract supplementation was significantly lower than the HFD group. ${ }^{33,34}$ Notably, White et al. showed that the epididymal and retroperitoneal adipose tissue weight were higher in the HFD group when compared to the SD group, ${ }^{31}$ demonstrating the effectiveness of the diets used.

As far as we know, no study has evaluated the relationship between the weight of brown adipose tissue, retroperitoneal adipose tissue, and the pancreas with eugenol clove oil supplementation. According to our results, eugenol supplementation did not improve the lipid profile. The HFDE group showed higher values of cholesterol and HDL, but the increase in HDL can be explained by the increase in total cholesterol. The literature shows opposing results for lipid profile. ${ }^{33,34}$ Elbahy; Madkour; Abdel-Raheem conducted a study in hyperlipidemic rats showing that the administration of $250 \mathrm{mg} \mathrm{kg}^{-1}$ of eugenol was beneficial for the lipid profile, with a significant reduction of total cholesterol and triglycerides in the hyperlipidemic group with eugenol when compared to the group without supplementation. ${ }^{37}$

It remains unclear why triglyceride levels were lower in the HFD group when compared to the SD group, but in the literature we found the same unexpected results with $\mathrm{C} 57 \mathrm{BL} / 6 \mathrm{~N}$ mice. $^{38}$
Harb et al. also confirmed the effect of eugenol as hypolipidemic, and the authors suggested that this effect is mediated in part by the potential transient vanilloid receptor 1 (TRPV1). TRPV1 is expressed in tissues, organs, and sensory nerves and is involved in functional regulation in the liver, pancreas, adipose tissue, and also blood vessels. ${ }^{39}$ The findings showed that TRPV1 expression is positively regulated in the liver of hypercholesterolemic rats; however, the precise molecular mechanism by which eugenol influences TRPV1 is unclear. ${ }^{35}$ But in fact, activation of TRPV1 signaling promotes accumulation of fat, while inhibiting this signaling protects against fat accumulation. ${ }^{40}$

Our study showed no difference in insulin levels among the experimental groups. Jung et al. found that insulin levels in the HFD group were higher than in HFD with clove extract supplement, suggesting that the increased levels were reduced with supplementation. ${ }^{33}$ Therefore, our findings do not agree with this previous result. Another study by Srinivasan et al., administered eugenol intragastrical in doses of $2.5 \mathrm{mg}, 5 \mathrm{mg}$, and $10 \mathrm{mg} \mathrm{kg} \mathrm{kg}^{-1}$ in diabetic Wistar rats for 30 days. The authors showed that $10 \mathrm{mg}$ supplementation promoted a significant reduction in blood glucose levels, an increase in plasma insulin levels, and an improvement in body weight and hepatic glycogen content. ${ }^{41}$

Histological analysis demonstrated that eugenol had a possible protective effect on the liver, our findings confirm the results of Jung et al., Ding et al., Harb et al., and Jo et al., who found that eugenol or clove oil supplementation led to reduced fat droplets both in size and number. ${ }^{33-36}$ This effect might be explained by the role played by eugenol in decreasing the accumulation of hepatic lipid by modulating/activating calmodulin dependent protein kinase kinase (CAMKK) - AMPactivated protein kinase (AMPK) - sterol regulatory elementbinding protein 1 (SREBP1) signaling pathway, as well as the regulation of lipolysis-related genes such as a cluster of differentiation 36 (CD36), carnitine palmitoyltransferase-1 (CPT-1) and acyl-CoA oxidase (ACO). ${ }^{36}$

Finally, regarding the gut microbiota profile, eugenol supplementation increased the abundance of the Firmicutes phylum in both HFD and SD groups. This phylum contains more than 250 genera, being considered the largest bacterial phylum. Some of their genera are Bacillus, Lactobacillus, Mollicutes, and Clostridium. ${ }^{42,43}$ Although Firmicutes are not considered beneficial due to their capacity to absorb calories from the diet and increase fat storage in the body, ${ }^{32}$ currently it is already known that within this phylum, some beneficial genera can be found such as Lactobacillus with immunomodulatory activity ${ }^{44}$ and Clostridium leptum with anti-inflammatory actions. ${ }^{45}$ Therefore, the exact bacterial species that make up this phylum must be better analyzed. In Bacteroidetes phylum, eugenol supplementation also increased the abundance of its groups. This phylum similarly to Firmicutes, can also have beneficial or harmful effects. Beneficially, it can play a role in the prevention of obesity and preventing colonization of the gastrointestinal tract by pathogenic agents, but on the other hand, it can also act as pathogenic and be involved in serious diseases and infections. ${ }^{46}$ 
In the third phylum analyzed, Actinobacteria, it was observed that supplementation also increased the abundance in the groups without eugenol, which does not agree with Ma et al., who found that the HFD and HFD with AEE groups decreased the abundance of Actinobacteria. ${ }^{32}$ This Gram-positive phylum, has a large diversity and many beneficial species with probiotic potential. It is divided into six classes: Actinobacteria, Acidimicrobiia, Coriobacteriia, Nitriliruptoria, Rubrobacteria, and Thermoleophilia, ${ }^{47,48}$ which together with the Proteobacteria represent only $10 \%$ of relative abundance in our gut microbiota. Although groups of Actinobacteria phylum represent a small percentage, they are essential in the development and maintenance of intestinal homeostasis. This phylum is supposed to be involved in the modulation of intestinal permeability, metabolism, immune system, and the gut-brain axis. In addition, some bacterial genera, especially Bifidoabcterium, play a modulating and therapeutic role in intestinal diseases such as irritable bowel syndrome, colorectal cancer, ulcerative colitis, among others, and also in extra-intestinal diseases, non-alcoholic liver steatosis, depression, respiratory pathologies, psoriasis, among others. ${ }^{49}$ Other beneficial effects of this phylum are being sources of new antibiotics, antifungals, and anticancer agents. ${ }^{47}$

The last analyzed phylum, Proteobacteria, was increased in the SDE group when compared to the SD group, and it was the only phylum whose abundance was reduced by eugenol supplementation in HFD even without a statistical difference. This may suggest a beneficial result since Proteobacteria has been recognized as producing metabolic endotoxemia (ME), that is, a change in permeability due to increased lipopolysaccharides (LPS). ${ }^{50}$ This ME drives the inflammatory process, triggers weight gain and can lead to diabetes onset. ${ }^{51}$ Besides, this phylum has also been associated with inflammation of the airways and the increase in its abundance causes an ecological imbalance, leading to greater severity of bronchiectasis. ${ }^{52}$

In addition to these harmful effects, according to some authors, Proteobacteria may represent a "microbial signature" of disease ${ }^{53}$ and also is considered as pathogenic with inflammatory potential, which may be the source of increased LPS levels in the visceral adipose tissue, ${ }^{54}$ and the proliferation of this phylum in the gut is associated with dysbiosis or reduced diversity of the intestinal microbial community. ${ }^{55}$

These Gram-negative bacteria, have the largest phylogenetic composition (116 bacterial families), and the phylum is divided into 6 classes: Alphaproteobacteria, Betaproteobacteria, Gammaproteobacteria, Deltaproteobacteria, Epsilonproteobacteria, and Zetaproteobacteria. ${ }^{53,55}$ To our knowledge, no further studies were found investigating eugenol and/or clove oil supplementation in mice fed with HFD. However, the antimicrobial activity of eugenol is already well established in the literature, ${ }^{24,25,56,57}$ although there are no studies that relate it to the gut microbiota of obese animals and/or humans. Therefore, more studies are required to elucidate the possible mechanisms involved in this outcome.

The limitations of the present study are related to the difficulty in finding studies with eugenol itself; therefore, those who administered clove extract were also used to discuss some variables. Another important limitation was regarding the dose administered. Despite being proved to be within a safe and nontoxic dosage, Harb et al. showed that eugenol in lower doses exhibited greater efficacy and better results in body and liver weight, and lipid profile using doses of eugenol at $10 \mathrm{mg}$ and $100 \mathrm{mg}$ associated with HFD in only 4 weeks of treatment. ${ }^{35}$

\section{Conclusion}

Eugenol supplementation appeared to prevent liver fat accumulation in the HFDE group. Besides, eugenol supplementation seemed to improve the profile of gut microbiota by promoting both an increase in the Actinobacteria phylum and a reduction in the phylum Proteobacteria. On the other hand, it did not promote beneficial effects related to metabolic profile or weight gain prevention in mice fed a high-fat diet. The implication of these findings on humans still needs to be investigated in further research.

\section{Abbreviations}

ACO Acyl-CoA oxidase

AEE Aspirin ester with eugenol

AMPK AMP-activated protein kinase

ANOVA Analysis of variance

AUC Area under the curve

CAMKK Calmodulin dependent protein kinase kinase

CD Chronic disease

CD36 Cluster of differentiation 36

CPT-1 Carnitine palmitoyltransferase-1

CT Cycle threshold

FC Fold change

FP Forward primer

GM Gut microbiota

GTT Glucose tolerance test

HCD Hypercholesterolemic diet

HFD High-fat diet

HFDE High-fat diet with eugenol

ME Metabolic endotoxemia

RP Reverse primer

SD Standard diet

SDE Standard diet with eugenol

SE $\quad$ Standard error

SREBP1 Sterol regulatory element-binding protein 1

TRPV1 Transient receptor potential cation channel subfamily $\mathrm{V}$ member 1

\section{Ethics statements}

All mice experiments were performed in accordance with the ARRIVE guidelines and the ethical principles had their origins in the Declaration of Helsinki in the U.K. Animals (Scientific Procedures). 


\section{Author contributions}

Marina Rodrigues: conceptualization, methodology, formal analysis, investigation, writing - original draft, writing - review and editing, visualization, project administration, funding acquisition. Caroline Bertoncini-Silva: methodology, writing original draft, writing - review and editing, visualization. Anderson Gregorio Joaquim: formal analysis. Cássia Dias Machado: methodology. Leandra Náira Zambelli Ramalho: resources. Daniela Carlos: resources. Priscila Giacomo Fassini: writing - review \& editing. Vivian Marques Miguel Suen: conceptualization, methodology, writing - original draft, writing review and editing, project administration.

\section{Conflicts of interest}

The authors declare no conflict of interest.

\section{Acknowledgements}

We thank Deisy M. da Silva for assistance with histological analyses and Jhefferson Barbosa Guimarães for the assistance with lipid profile and gut microbiota. This work was supported by the Coordenação de Aperfeiçoamento de Pessoal de Nível Superior (CAPES).

\section{References}

1 A. R. O. Pinheiro, S. F. T. Freitas and A. C. T. Corso, Uma abordagem epidemiológica da obesidade, Rev. Nutr., 2004, 17, 523-533, DOI: 10.1590/S1415-52732004000400012.

2 Associação Brasileira para o Estudo da Obesidade e da Síndrome Metabólica, Diretrizes brasileiras de obesidade 4 edn, 2016. Available in: https://abeso.org.br/wp-content/ uploads/2019/12/Diretrizes-Download-Diretrizes-Brasileirasde-Obesidade-2016.pdf.

3 E. N. Wanderley and V. A. Ferreira, Obesidade: uma perspectiva plural, Cien. Saude Colet., 2010, 15, 185-194, DOI: 10.1590/S1413-81232010000100024.

4 World Health Organization, Obesity and overweight, 2020. Available in: https:/www.who.int/news-room/fact-sheets/ detail/obesity-and-overweight.

5 R. L. Soeiro, G. S. C. Valente, E. A. Cortez, L. M. Mesquita, S. C. M. Xavier and B. M. I. S. Lobo, Educação em saúde em grupo no tratamento de obesos grau III: Um desafio para os profissionais de saúde, Rev. Bras. Educ. Med., 2019, 43, 681-691, DOI: 10.1590/1981-5271v43suplemento120190005..

6 N. Steckhan, C. D. Hohmann, C. Kessler, G. Dobos, A. Michalsen and H. Cramer, Effects of different dietary approaches on inflammatory markers in patients with metabolic syndrome: A systematic review and meta-analysis, Nutrition, 2016, 32, 338-348, DOI: 10.1016/j. nut.2015.09.010.
7 J. K. Sethi and A. J. Vidal-Puig, Thematic review series: adipocyte biology. Adipose tissue function and plasticity orchestrate nutritional adaptation, J. Lipid Res., 2007, 48, 1253-1262, DOI: 10.1194/jlr.R700005-JLR200.

8 A. B. Mariath, L. P. Grillo, R. O. da Silva, P. Schmitz, I. C. de Campos, J. R. P. Medina and R. M. Kruger, Obesidade e fatores de risco para o desenvolvimento de doenças crônicas não transmissíveis entre usuários de unidade de alimentação e nutrição, Cad. Saúde Pública, 2007, 23, 897905, DOI: 10.1590/S0102-311X2007000400017.

9 P. Kopelman, Health risks associated with overweight and obesity, Obes. Rev., 2007, 1, 13-17, DOI: 10.1111/j.1467789X.2007.00311.x.

10 E. Lau, D. Carvalho, C. Pina-Vaz, J. A. Barbosa and P. Freitas, Beyond gut microbiota: Understanding obesity and type 2 diabetes, Hormones, 2015, 14, 358-369, DOI: 10.14310/horm.2002.1571.

11 N. Arslan, Obesity, fatty liver disease and intestinal microbiota, World J. Gastroenterol., 2014, 20, 16452-16463, DOI: 10.3748/wjg.v20.i44.16452.

12 E. Amabebe, F. O. Robert, T. Agbalalah and E. S. F. Orubu, Microbial dysbiosis-induced obesity: Role of gut microbiota in homoeostasis of energy metabolism, Br. J. Nutr., 2020, 123, 1127-1137, DOI: 10.1017/S0007114520000380.

13 N. Kobyliak, O. Virchenko and T. Falalyeyeva, Pathophysiological role of host microbiota in the development of obesity, Nutr. J., 2016, 15, DOI: 10.1186/s12937016-0166-9.

14 D. Knights, K. G. Lassen and R. J. Xavier, Advances in inflammatory bowel disease pathogenesis: Linking host genetics and the microbiome, Gut, 2013, 62, 1505-1510, DOI: 10.1136/gutjnl-2012-303954.

15 L. Flandroy, T. Poutahidis, G. Berg, G. Clarke, M. C. Dao, E. Decaestecker, E. Furman, T. Haahtela, S. Massart, H. Plovier, Y. Sanz and G. Rook, The impact of human activities and lifestyles on the interlinked microbiota and health of humans and of ecosystems, Sci. Total Environ., 2018, 627, 1018-1038, DOI: 10.1016/j.scitotenv.2018.01.288.

$16 \mathrm{~S}$. Sirisinha, The potential impact of gut microbiota on your health: Current status and future challenges, Asian Pac. J. Allergy Immunol., 2016, 34, 249-264, DOI: 10.12932/ AP0803.

17 S. M. Jandhyala, R. Talukdar, C. Subramanyam, H. Vuyyuru, M. Sasikala and D. N. Reddy, Role of the normal gut microbiota, World J. Gastroenterol., 2015, 21, 8787-8803, DOI: 10.3748/wjg.v21.i29.8787.

18 R. E. Ley, P. J. Turnbaugh, S. Klein and J. I. Gordon, Human gut microbes associated with obesity, Nature, 2006, 444, 1022-1023, DOI: 10.1038/4441022a.

19 R. E. Ley, F. Bäckhed, P. Turnbaugh, C. A. Lozupone, R. D. Knight and J. I. Gordon, Obesity alters gut microbial ecology, Proc. Natl. Acad. Sci. U. S. A., 2005, 102, 1107011075, DOI: 10.1073/pnas.0504978102.

20 J. Miyamoto, M. Igarashi, K. Watanabe, S. I. Karaki, H. Mukouyama, S. Kishino, X. Li, A. Ichimura, J. Irie, Y. Sugimoto, T. Mizutani, T. Sugawara, T. Miki, J. Ogawa, 
D. J. Drucker, M. Arita, H. Itoh and I. Kimura, Gut microbiota confers host resistance to obesity by metabolizing dietary polyunsaturated fatty acids, Nat. Commun., 2019, 10, 4007, DOI: 10.1038/s41467-019-11978-0.

21 L. M. de Freitas Junior and E. B. de Almeida Jr., Medicinal plants for the treatment of obesity: Ethnopharmacological approach and chemical and biological studies, Am. J. Transl. Res., 2017, 9, 2050-2064.

22 R. Scherer, R. Wagner, M. C. T. Duarte and H. T. Godoy, Composição e atividades antioxidante e antimicrobiana dos óleos essenciais de cravo-da-índia, citronela e palmarosa, Rev. Bras. Plant. Med., 2009, 11, 442-449, DOI: 10.1590/ S1516-05722009000400013.

23 P. Mazzafera, Efeito alelopático do extrato alcoólico do cravo-da-índia e eugenol, Rev. Bras. Bot., 2003, 26, 231-238, DOI: $10.1590 /$ S0100-84042003000200011.

24 R. S. Affonso, M. N. Rennó, G. B. C. A. Slana and T. C. C. França, Aspectos químicos e biológicos do óleo essencial de cravo da índia, Rev. Virtual Quim., 2012, 4, 146-161, DOI: 10.5935/1984-6835.20120012.

25 R. A. Oliveira, T. V. Reis, C. K. Sacramento, L. P. Duarte and F. F. Oliveira, Constituintes químicos voláteis de especiarias ricas em eugenol, Rev. Bras. Farmacogn., 2009, 19, 771775, DOI: 10.1590/S0102-695X2009000500020.

26 M. Wlodarska, B. P. Willing, D. M. Bravo and B. B. Finlay, Phytonutrient diet supplementation promotes beneficial Clostridia species and intestinal mucus secretion resulting in protection against enteric infection, Sci. Rep., 2015, 5, 9253, DOI: 10.1038/srep09253.

27 C. B. Silva, P. G. Fassini, L. N. Z. Ramalho, E. C. da Conceição, A. J. C. M. Zordan, D. Carlos and V. M. M. Suen, Curcuma supplementation in high-fat-fed C57BL/6 mice: No beneficial effect on lipid and glucose profile or prevention of weight gain, Eur. J. Nutr., 2020, 59, 93-102, DOI: 10.1007/s00394-018-1887-7.

28 A. J. C. M. Zordan, C. B. Silva, A. G. Joaquim, M. Rodrigues, C. D. Machado, D. Carlos, M. C. F. Freitas and V. M. M. Suen, Gingerol supplementation does not change glucose tolerance, lipid profile and does not prevent weight gain in C57BL/6 mice fed a high-fat diet, Clin. Nutr. Exp., 2020, 32, 11-19, DOI: 10.1016/j.yclnex.2020.05.001.

29 M. C. G. Miranda, R. P. Oliveira, L. Torres, S. L. F. Aguiar, N. Pinheiro-Rosa, L. Lemos, M. A. Guimarães, D. Reis, T. Silveira, E. Ferreira, T. G. Moreira, D. C. Cara, T. U. Maioli, B. L. Kelsall, D. Carlos and A. M. C. Faria, Frontline Science: Abnormalities in the gut mucosa of nonobese diabetic mice precede the onset of type 1 diabetes, J. Leukocyte Biol., 2019, 106, 513-529, DOI: 10.1002/ JLB.3HI0119-024RR.

30 T. F. S. N. N. De Souza, Efeito antinociceptivo $e$ antiedematogênico do eugenol, Dissertação, Universidade Estadual do Ceará, 2007.

31 P. A. S. White, L. M. Cercato, J. M. D. Araújo, L. A. Souza, A. F. Soares, A. P. O. Barbosa, J. M. R. Neto, A. C. Marçal, U. F. Machado, E. A. Camargo, M. R. V. Santos and L. C. Brito, Modelo de obesidade induzida por dieta hiperlipídica e associada à resistência à ação da insulina e intolerância à glicose, Arq. Bras. Endocrinol. Metabol., 2013, 57, 339-345, DOI: 10.1590/S0004-27302013000500002.

32 N. Ma, X. W. Liu, X. J. Kong, S. H. Li, Z. H. Jiao, Z. Qin, Y. J. Yang and J. Y. Li, Aspirin eugenol ester regulates cecal contents metabolomic profile and microbiota in an animal model of hyperlipidemia, BMC Vet. Res., 2018, 14, 405, DOI: 10.1186/s12917-018-1711-x.

33 C. H. Jung, J. Ahn, T. I. Jeon, T. W. Kim and T. Y. Ha, Syzygium aromaticum ethanol extract reduces high-fat dietinduced obesity in mice through downregulation of adipogenic and lipogenic gene expression, Exp. Ther. Med., 2012, 4, 409-414, DOI: 10.3892/etm.2012.609.

34 Y. Ding, Z. Gu, Y. Wang, S. Wang, H. Chen, H. Zhang, W. Chen and Y. Q. Chen, Clove extract functions as a natural fatty acid synthesis inhibitor and prevents obesity in a mouse model, Food Funct., 2017, 8, 2847-2856, DOI: 10.1039/c7fo00096k.

35 A. A. Harb, Y. K. Bustanji, I. M. Almasri and S. S. Abdalla, Eugenol reduces ldl cholesterol and hepatic steatosis in hypercholesterolemic rats by modulating TRPV1 receptor, Sci. Rep., 2019, 9, 14003, DOI: 10.1038/s41598-01950352-4.

36 H. K. Jo, G. W. Kim, K. J. Jeong, D. Y. Kim and S. H. Chung, Eugenol ameliorates hepatic steatosis and fibrosis by down-regulating SREBP1 gene expression via AMPK-mTORp70S6 K signaling pathway, Biol. Pharm. Bull., 2014, 37, 1341-1351, DOI: 10.1248/bpb.b14-00281.

37 D. A. Elbahy, H. I. Madkour and M. H. Abdel-Raheem, Evaluation of antihyperlipidemic activity of eugenol in triton induced hyperlipidemia in rats, Int. J. Res. Stud. Biosci., 2015, 3, 19-26.

38 C. Podrini, E. L. Cambridge, C. J. Lelliott, D. M. Carragher, J. Estabel, A. K. Gerdin, N. A. Karp, C. L. Scudamore, Sanger Mouse Genetics Project, R. Ramirez-Solis and J. K. White, High-fat feeding rapidly induces obesity and lipid derangements in $\mathrm{C} 57 \mathrm{BL} / 6 \mathrm{~N}$ mice, Mamm. Genome, 2013, 24, 5-6, DOI: 10.1007/s00335-013-9456-0.

39 P. Wang, D. Liu and Z. Zhu, Transient Receptor Potential Vanilloid Type-1 Channel in Cardiometabolic Protection, J. Korean Soc. Hypertens., 2011, 17, 37-47, DOI: 10.5646/ jksh.2011.17.2.37.

40 A. L. Motter and G. P. Ahern, TRPV1-null mice are protected from diet-induced obesity, FEBS Lett., 2008, 582, 2257-2262, DOI: 10.1016/j.febslet.2008.05.021.

41 S. Srinivasan, G. Sathish, M. Jayanthi, J. Muthukumaran, U. Muruganathan and V. Ramachandran, Ameliorating effect of eugenol on hyperglycemia by attenuating the key enzymes of glucose metabolism in streptozotocin-induced diabetic rats, Mol. Cell. Biochem., 2014, 385, 159-168, DOI: 10.1007/s11010-013-1824-2.

42 M. Bajzer and R. J. Seeley, Physiology: obesity and gut flora, Nature, 2006, 444, 1009-1010, DOI: 10.1038/4441009a.

43 H. Tilg, A. R. Moschen and A. Kaser, Obesity and the microbiota, Gastroenterologia, 2009, 136, 1476-1483, DOI: 10.1053/j.gastro.2009.03.030. 
44 J. O'Callaghan and P. W. O'Toole, Lactobacillus: hostmicrobe relationships, Curr. Top. Microbiol. Immunol., 2013, 358, 119-154, DOI: 10.1007/82_2011_187.

45 J. Kabeerdoss, V. Sankaran, S. Pugazhendhi and B. S. Ramakrishna, Clostridium leptum group bacteria abundance and diversity in the fecal microbiota of patients with inflammatory bowel disease: a case-control study in India, BMC Gastroenterol., 2013, 13, DOI: 10.1186/1471230X-13-20.

46 H. M. Wexler, Bacteroides: The good, the bad, and the nitty-gritty, Clin. Microbiol. Rev., 2007, 20, 593-621, DOI: 10.1128/CMR.00008-07.

47 E. A. Barka, P. Vatsa, L. Sanchez, N. Gaveau-Vaillant, C. Jacquard, H.-P. Klenk, C. Clément, Y. Ouhdouch and G. P. V. Wezel, Taxonomy, Physiology, and Natural Products of Actinobacteria, Microbiol. Mol. Biol. Rev., 2015, 80, 1-43, DOI: 10.1128/MMBR.00019-15.

48 B. Gao and R. S. Gupta, Phylogenetic framework and molecular signatures for the main clades of the phylum Actinobacteria, Microbiol. Mol. Biol. Rev., 2012, 76, 66-112, DOI: 10.1128/MMBR.05011-11.

49 C. Binda, L. R. Lopetuso, G. Rizzatti, G. Gibiino, V. Cennamo and A. Gasbarrini, Actinobacteria: A relevant minority for the maintenance of gut homeostasis, Dig. Liver Dis., 2018, 50, 421-428, DOI: 10.1016/j.dld.2018.02.012.

50 C. J. Chang, C. S. Lin, C. C. Lu, J. Martel, Y. F. Ko, D. M. Ojcius, S. F. Tseng, T. R. Wu, Y. Y. Chen, J. D. Young and H. C. Lai, Ganoderma lucidum reduces obesity in mice by modulating the composition of the gut microbiota, Nat. Commun., 2015, 6, DOI: 10.1038/ncomms8489.

51 P. D. Cani, J. Amar, M. A. Iglesias, M. Poggi, C. Knauf, D. Bastelica, A. M. Neyrinck, F. Fava, K. M. Tuohy, C. Chabo, A. Waget, E. Delmée, B. Cousin, T. Sulpice, B. Chamontin, J. Ferrières, J. F. Tanti, G. R. Gibson,
L. Casteilla, N. M. Delzenne, M. C. Alessi and R. Burcelin, Metabolic endotoxemia initiates obesity and insulin resistance, Diabetes, 2007, 56, 1761-1772, DOI: 10.2337/db061491.

52 W. J. Guan, J. J. Yuan, H. M. Li, Y. H. Gao, Y. Huang, C. L. Chen, R. C. Chen and N. S. Zhong, Proteobacteria community compositions correlate with bronchiectasis severity, Int. J. Tuberc. Lung Dis., 2018, 22, 1095-1105, DOI: 10.5588/ ijtld.18.0037.

53 G. Rizzatti, L. R. Lopetuso, G. Gibiino, C. Binda and A. Gasbarrini, Proteobacteria: A Common Factor in Human Diseases, BioMed Res. Int., 2017, DOI: 10.1155/2017/ 9351507.

54 M. M. Pérez, L. M. S. Martins, M. S. Dias, C. A. Pereira, J. A. Leite, E. C. S. Gonçalves, P. Z. de Almeida, E. N. de Freitas, R. C. Tostes, S. G. Ramos, M. R. de Zoete, B. Ryffel, J. S. Silva and D. Carlos, Interleukin-17/interleukin-17 receptor axis elicits intestinal neutrophil migration, restrains gut dysbiosis and lipopolysaccharide translocation in high-fat diet-induced metabolic syndrome model, Immunology, 2019, 156, 339-355, DOI: 10.1111/imm.13028.

55 N.-R. Shin, T. W. Whon and J.-W. Bae, Proteobacteria: microbial signature of dysbiosis in gut microbiota, Trends Biotechnol., 2015, 33, 496-503, DOI: 10.1016/j. tibtech.2015.06.011.

56 S. M. Nejad, H. Özgüneş and N. Başaran, Pharmacological and Toxicological Properties of Eugenol, Turk. J. Pharm. Sci., 2017, 14, 201-206, DOI: 10.4274/tjps.62207.

57 S. M. Ali, A. A. Khan, I. Ahmed, M. Musaddiq, K. S. Ahmed, H. Polasa, L. V. Rao, C. M. Habibullah, L. A. Sechi and N. Ahmed, Antimicrobial activities of eugenol and cinnamaldehyde against the human gastric pathogen Helicobacter pylori, Ann. Clin. Microbiol. Antimicrob., 2005, 4, DOI: 10.1186/1476-0711-4-20. 